\title{
Investigation of the impact of main control room digitalization on operators cognitive reliability in nuclear power plants
}

\author{
Yong Zhou ${ }^{\mathrm{a},}{ }^{*}$, HaiYing Mu ${ }^{\mathrm{a}}$, Jianjun Jiang ${ }^{\mathrm{c}}$ and Li Zhang ${ }^{\mathrm{b}}$ \\ ${ }^{a}$ Flight Technology college, Civil Aviation Flight University of China, 618307, Guanghan, China \\ ${ }^{\mathrm{b}}$ Human Factors Institute, University of South China, 421001, Hengyang, China \\ ${ }^{\mathrm{c}}$ Department of Computer Engineering, KangDa Vocational Technical College, 511363, Guangzhou, China
}

\begin{abstract}
Currently, there is a trend in nuclear power plants (NPPs) toward introducing digital and computer technologies into main control rooms (MCRs). Safe generation of electric power in NPPs requires reliable performance of cognitive tasks such as fault detection, diagnosis, and response planning. The digitalization of MCRs has dramatically changed the whole operating environment, and the ways operators interact with the plant systems. If the design and implementation of the digital technology is incompatible with operators' cognitive characteristics, it may have negative effects on operators' cognitive reliability. Firstly, on the basis of three essential prerequisites for successful cognitive tasks, a causal model is constructed to reveal the typical human performance issues arising from digitalization. The cognitive mechanisms which they impact cognitive reliability are analyzed in detail. Then, Bayesian inference is used to quantify and prioritize the influences of these factors. It suggests that interface management and unbalanced workload distribution have more significant impacts on operators' cognitive reliability.
\end{abstract}

Keywords: digital main control room, cognitive reliability, human factors issues, human errors, bayesian network

\section{Introduction}

Main control rooms (MCRs) are crucial stations for supervising and controlling the nuclear power plants (NPPs). Conventional MCRs are dominated by analog equipments, such as electromechanical switches, panel-mounted meters, annunciators, chart recorders, etc. Nowadays, with the progress of computer and digital technologies, analog equipments are being replaced with modern digital human system interfaces (HSIs), including computer-based information display system, computer-based soft control system, computerized operating procedures, intelligent decision support system, and so on.

The use of advanced technology is generally considered to improve the safety of NPPs, however, if poorly designed and implemented, it may have the potential to affect human performance and reduce human reliability [11]. The MCRs and HSIs digitali- zation has dramatically changed the whole operating environment, the role of operators, and the ways they interact with the systems. The objective of this research is to investigate critical human performance problems arising from digitalization, analyze the cognitive mechanisms which they impact operators' cognitive reliability, and measure the effects of them.

\section{Theoretical basis for the model}

From an information-processing view, human cognition can be conceptualized as a series of mental operation process which is based on received information. Any variation, failing, or weakness in these mental operations will impact human cognitive reliability. Norman and Bobrow characterized human cognitive processes as being limited by either the

\footnotetext{
"Corresponding author. Tel.:+86 15181049689; fax: +86 08385182124; E-mail: zhouyongm@yahoo.cn.
} 
adequacy of available cognitive resources or the quality of available data [10].

The availability of cognitive resources determined by factors such as attention bandwidth, memory capacity, perception and response channels, and so on. Operators' tasks often require concurrent mental operations, and each operation requires some cognitive resources. When resources demands exceed the available supply, the task performance will degrade. In this situation, the cognitive process is called resource-limited.

The availability and quality of data is determined by what is being processed. When there is a lack of necessary information, performance also will be limited. In this situation, the cognitive process is called data-limited. It can be further subdivided into signal data limits and memory data limits. Signal data limit means operators can't get enough required information from the human-machine interface or environment. It may result from poor interface design or low signal-to-noise ratio, etc. Memory data limit does not describe the amount of memory, but the nature of knowledge stored in the memory, as the quality of those stored knowledge influence the processing of new information.

Human error can be explained on the basis of a relatively small number of cognitive elements and mechanism $[15,16]$. According to above cognitive processing limits theory, operators' cognition reliability mainly depends on the availability of cognitive resources, the availability and quality of necessary control information about the NPPs, and the correct- ness and completeness of knowledge or schema stored in operators' memory. The mechanism which MCRs digitalization can affect human reliability is chiefly through them.

\section{Construction of the causal model}

Through interview, field investigation, and literature review, typical human factor issues arising from digitalization have been identified. They include increased cognitive workload due to interface management task, difficulty in understanding how systems work due to increased complexity and opacity, loss of vigilance and skill due to high level of automation, difficulty in navigating through computerbased information system, and so on. These factors may tax human cognitive resources, corrupt operators' schema or mental model, and negatively impact their information acquisition activities. Fig. 1 shows their cause and effect relationships.

\subsection{Factors affecting the quality of information acquisition}

\subsubsection{Inappropriate trust in automation}

Operators' reliance or trust level in automation systems is a very important human-machine relationship. It determines operators' attitudes toward the automations. Operators tend to trust more reliable, robust, familiar, and predictable systems [18].

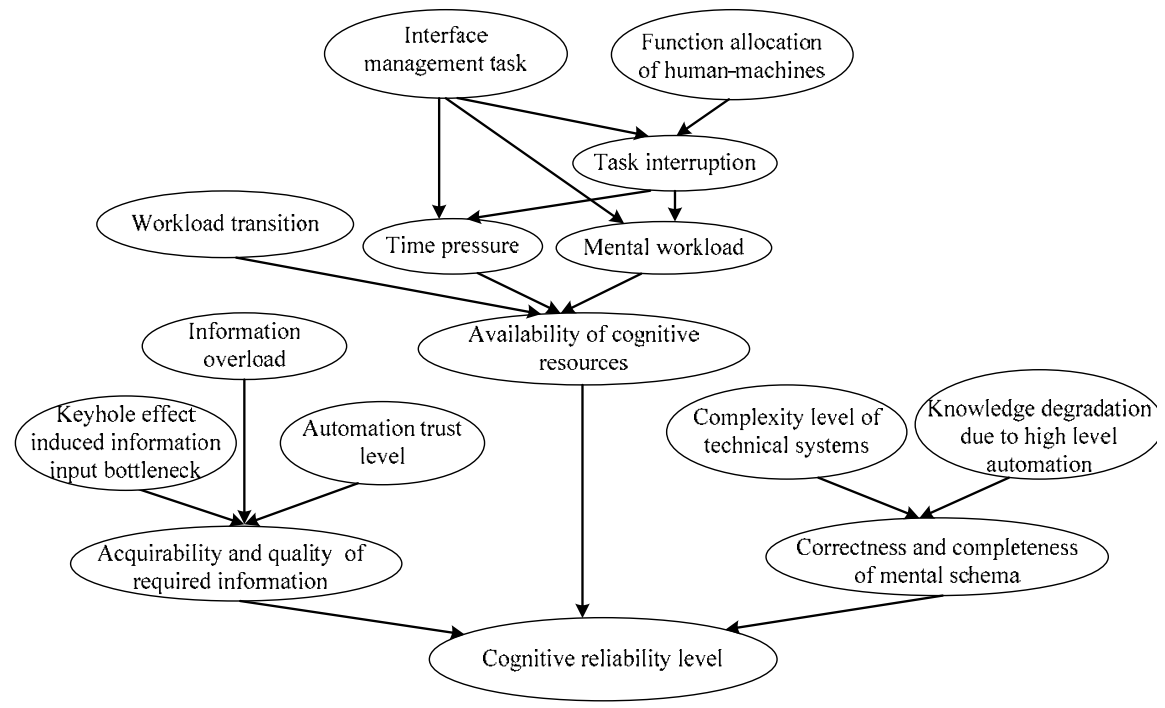

Figure 1. A causal model of factors influencing cognitive reliability in digital MCRs 
In digital MCR, there exist factors which can both increase and decrease operators' trust level. For example, the opacity and complexity of the systems will degrade operators' trust level, but the high reliability of the computer and digital systems will enhance it, and the latter becomes more obvious.

Inappropriate automation trust can make operators omit the necessary information. Over-trust or overreliance on the automated systems can result in vigilance decrements, and make operator act as a passive monitor $[5,14]$. On the contrary, operators who have under-trust in automation tend to falsely reject information, even when the information is correct and important.

\subsubsection{Keyhole effect}

In conventional MCRs, there are many spatially dedicated indicators and displays. Each of them has fixed locations. Operators can physically navigate to plant information by shifting their attention. In digital MCRs, information about the plant is made available to operators only through the computerized HSIs. An important property of the computer-based display systems is that the viewport size is very small relative to the large size of the data space. Most of the information is hidden from view. The amount of information that can be accessed at one time is extremely small. This property has been referred to as the keyhole effect [21]. The consequence of the keyhole effect is that it restrict operators' perceptual field, and impair their ability to perceive plants' information.

\subsubsection{Information overload}

In digital MCRs, advanced technologies have greatly increased the ability to collect, transmit, and transform data. Computer-based information systems can generate and display various data in detail, such as process control data, maintenance and engineering data, configuration data, and so on. Although operators can get various data from the information systems, they must find and choose the required information from a large amount of information. Operators often feel overloaded by the overwhelming amount of information. Information overload poses a great challenge for information intake. As human can only take in a limited amount of information at a time, when the amount of presented information are far beyond the eye and brain can handle it, significant lapses in attention or information omission errors will occur.

\subsection{Factors affecting the correctness and completeness of cognitive schema}

\subsubsection{Increased Complexity of Technical System}

Operators' cognitive schema is the internal representation of the physical and functional characteristics of the plant system and its operation, such as physical appearance and layout of control panel, causal relations among plant's components, systems dynamics, how systems operate, and so on. The more complex the system is, the more difficult the schema construction becomes. Compared with conventional MCRs, the digital MCRs appear to be more simple and compact, but in reality they have become more complex. The introduction of new technologies such as intelligent agents, information processing support, and software-mediated interface, etc, not only increase the number of components throughout the plant, but also make the opacity and coupling of the systems to the highest level. There are hundreds of subsystems which interact under different situations. If the schema in operators' mind is incomplete or has fault, then their interpretation and diagnosis about the plant's state will be wrong.

\subsubsection{Knowledge and skill degradation due to high- level automation}

Operators' cognitive schema is constructed and updated mainly through formal education, special training, and accumulation of operational experience. The adoption of high level of automation can assist the operators and minimize their workload, but they also have negative effects. Firstly, operators who control the highly automated systems often have a limited knowledge and understanding of systems' behavior [19]. Operators may not actively try to construct the schema of the automation systems due to overreliance. Secondly, many tasks and functions once performed by operators have now been highly automated. Operators' schema becomes as inert knowledge, and can not maintain its correctness and completeness due to the lack of opportunities to practice and renew.

\subsection{Factors influencing availability of cognitive resources}

\subsubsection{Mental workload transitions}

In digital MCRs, advanced automation and computer systems can execute the greater part of the tasks. The role of operator has changed from an active controller to a passive observer. During normal operating 
situation, operators may face prolonged periods of low workload. However, in accident conditions, especially when operator must simultaneously take up manual control due to automation failure, their workload may jump sharply.

The sudden transitions between extreme low workload and critical high workload can cause cognitive cost and performance degradation. Firstly, the low workload state which immediately subsequent to the high workload state, may make the short-term memory buffer continue to be overloaded even after the workload shift downward [3]. Secondly, sudden workload transition can induce psychological stress, and result in shrinkage of cognitive resources. Thirdly, although operators are capable of maintaining performance across workload transitions by using various adaptive methods such as effort regulation or changing resource allocation strategy $[4,20]$, adaptation to workload transitions itself will consume mental resources, and may result in fatigue after-effects. Besides, the inappropriate resource allocation strategy can also reduce the available cognitive resources.

\subsubsection{Interface management tasks}

The primary tasks performed by nuclear power plant operators are process monitoring and control. However, in digital MCRs, HSIs are computer-based. Operators can not directly interact with the plant anymore. To perform the monitoring and control task, operators must actively interact with the computerized interface, and need to perform interface management tasks including searching for data, navigating through displays, configuring interfaces, scaling windows, etc.

The interface management tasks can impose additional mental burden on operators. Firstly, managing the interface can increase operators' workload. According to multiple resources model [22], the interface management tasks and the primary tasks often rely on the same type of cognitive resources such as the same sensory channel and processing structure. Interface management draw cognitive resources away from the primary task and thereby make it resource limited. Secondly, interface management tasks often distract operators' attention, and interfere with their limited memory buffer. The distraction or interruption may cause waste of cognitive resources. Thirdly, since the allowed response time under accident conditions is limited, managing the interface can increase operator's time pressure, and finally lead to attention narrowing or reduction in working memory capacity [12].

\subsubsection{Task interruption}

The Task interruption widely exists in the multitasking environment [1]. In digital MCRs, this problem becomes more serious. Firstly, there are more competing tasks resulting from additional interface management. Managing the interface shall break the continuity of cognitive focus on the primary task. Operators must often suspend one task so that another task can be performed. Secondly, the inappropriate allocation of human-machine function can also cause task interruption. One of the main trends in MCRs design is an increase in automation of tasks which traditionally performed by the operator [13]. If the implementation of automation technology does not consider whether a function should be automated with respect to operator's capability, their tasks will be poorly organized and lacking consistency.

Interruption diverts operator's attention from ongoing task, forces him to turn his attention towards the interruption, and then refocuses on the previous task. Switching among multiple concurrent tasks may consume a mass of attention resources. Interruption also creates prospective memory demands because individual must remember to resume the interrupted task after the end of the interruption [2]. Besides, task interruption and task switching can result in extra time cost [9]. Operator may experience a greater feeling of time pressure.

\section{Quantitative Analysis of the Causal Model by Using Bayesian Network}

\subsection{Bayesian networks}

Bayesian network, also is known as Bayesian Belief Network, belongs to the family of probabilistic graphical models [6]. Consider a Bayesian network over $U=\left\{X_{1}, \ldots, X_{n}\right\}$, where $X_{1}, \ldots, X_{n}$ represent nodes. Then the joint probability distribution $P(U)$ is the product of all potentials specified in $U$ [7].

$$
P(U)=\prod_{i=1}^{n} P\left(X_{i} \mid p a\left(X_{i}\right)\right)
$$

Where $p a\left(X_{i}\right)$ are the parents of node $X_{i}$. The marginal probability of $X_{i}$ is:

$$
P\left(X_{i}\right)=\sum_{\text {except } X_{i}} P(U)
$$


One of main applications of Bayesian network is as an inference engine for calculating the probability of an event given the observation of other events, called evidence. Assume an evidence e is found, we can calculate the probability of the occurrence of an event given some evidences through the following formula.

$$
P\left(X_{i} \mid e\right)=\frac{\sum_{U \backslash\left\{X_{i}\right\}} P(U, e)}{P(e)}
$$

Where $X \in U, U \backslash\left\{X_{i}\right\}$ represent the rest variables in $U$ except $X_{i}$, and

$$
P(U, e)=\prod_{i=1}^{n} P\left(X_{i} \mid p a\left(X_{i}\right)\right) \prod_{j=1}^{m} e_{j}
$$

\subsection{Estimation of the prior probability and conditional probability of each node}

Before conducting Bayesian inference, the prior probability or conditional probability of each node in Fig. 1 should be specified. Since the digitalization of MCRs has only been implemented for a short period of time, there is a lack of enough historical human error data. Here, the values of prior and conditional probabilities are obtained by using engineering judgment method. As it is often difficult for human to estimate the occurrence possibility of events by using single numerical probability, the fuzzy expression of their opinions about the probabilities of events is used. The advantage of using fuzzy probabilities is that it is more intuitive for experts to give their opinions, and can reduce judgment biases. For simplicity, triangular form membership function is adopted to describe all fuzzy probabilities.

Table 1 gives the fuzzy prior probabilities of root nodes in Fig. 1. As shown in Table 1, there are three substates for each of these nodes, for example, the substates which describe the severity of "workload transition (WT)" include "severe", "moderate", and "minor". The occurrence likelihood of them is defined by experts as a triangular fuzzy number such as $P\left(W T_{1}\right)=(0.06,0.10,0.14)$. Table 2 gives the conditional fuzzy probabilities of variable "task interruption frequency (TI)" given the states of nodes "interface management task (IM)" and "Function allocation of human-machines and task design $(F A)$ ".

Table 1

\begin{tabular}{|c|c|c|c|}
\hline Variables & \multicolumn{3}{|c|}{ Substates and probabilities } \\
\hline \multirow{3}{*}{ Workload transition $(W T)$} & $\operatorname{Severe}\left(W T_{1}\right)$ & Moderate $\left(W T_{2}\right)$ & $\operatorname{Minor}\left(W T_{3}\right)$ \\
\hline & $(0.06,0.10,0.14)$ & $(0.78,0.87,0.96)$ & $(0.02,0.03,0.04)$ \\
\hline & 0.10 & 0.87 & 0.03 \\
\hline \multirow{3}{*}{ Interface management $(I M)$} & Complicated $\left(I M_{1}\right)$ & Acceptable $\left(I M_{2}\right)$ & Simple $\left(I M_{3}\right)$ \\
\hline & $(0.08,0.12,0.16)$ & $(0.76,0.87,0.98)$ & $(0.009,0.01,0.011)$ \\
\hline & 0.12 & 0.87 & 0.01 \\
\hline \multirow{3}{*}{$\begin{array}{l}\text { Function allocation of } \\
\text { human-machines and task } \\
\text { design }(F A)\end{array}$} & Inappropriate $\left(F A_{1}\right)$ & Moderate $\left(F A_{2}\right)$ & Suitable $\left(F A_{3}\right)$ \\
\hline & $(0.01,0.04,0.07)$ & $(0.28,0.54,0.80)$ & $(0.36,0.42,0.48)$ \\
\hline & 0.04 & 0.54 & 0.42 \\
\hline \multirow{3}{*}{ Automation trust level $(A T)$} & Under-trust $\left(A T_{1}\right)$ & Adequate $\left(A T_{2}\right)$ & Over-trust $\left(A T_{3}\right)$ \\
\hline & $(0.02,0.05,0.08)$ & $(0.75,0.85,0.95)$ & $(0.08,0.10,0.12)$ \\
\hline & 0.05 & 0.85 & 0.10 \\
\hline \multirow{3}{*}{ Information overload $(I O)$} & $\operatorname{Serious}\left(I O_{1}\right)$ & Moderate $\left(\mathrm{IO}_{2}\right)$ & $\operatorname{Minor}\left(\mathrm{IO}_{3}\right)$ \\
\hline & $(0.05,0.08,0.11)$ & $(0.79,0.88,0.97)$ & $(0.02,0.04,0.06)$ \\
\hline & 0.08 & 0.88 & 0.04 \\
\hline \multirow{3}{*}{$\begin{array}{l}\text { Keyhole effect and infor- } \\
\text { mation acquisition bottle- } \\
\operatorname{neck}(K E)\end{array}$} & Severe $\left(K E_{1}\right)$ & Acceptable $\left(K E_{2}\right)$ & $\operatorname{Minor}\left(K E_{3}\right)$ \\
\hline & $(0.10,0.12,0.14)$ & $(0.79,0.86,0.93)$ & $(0.01,0.02,0.03)$ \\
\hline & 0.12 & 0.86 & 0.02 \\
\hline \multirow{3}{*}{$\begin{array}{l}\text { Level of complexity for } \\
\text { technical systems }(L C)\end{array}$} & $\operatorname{High}\left(L C_{1}\right)$ & $\operatorname{Medium}\left(L C_{2}\right)$ & $\operatorname{Low}\left(L C_{3}\right)$ \\
\hline & $(0.05,0.10,0.15)$ & $(0.78,0.87,0.96)$ & $(0.01,0.03,0.05)$ \\
\hline & 0.10 & 0.87 & $\mathbf{0 . 0 3}$ \\
\hline \multirow{3}{*}{$\begin{array}{l}\text { Knowledge degradations } \\
(K D)\end{array}$} & Severe $\left(K D_{1}\right)$ & Moderate $\left(K D_{2}\right)$ & $\operatorname{Minor}\left(K D_{3}\right)$ \\
\hline & $(0.02,0.10,0.18)$ & $(0.74,0.86,0.98)$ & $(0.02,0.04,0.06)$ \\
\hline & 0.10 & 0.86 & 0.04 \\
\hline
\end{tabular}

Fuzzy prior probabilities of root nodes 
Table 2

Conditional probability of variable "Task Interruption $(T I)$ " $P(T I \mid I M, F A)$

\begin{tabular}{|c|c|c|c|c|c|c|c|c|c|c|}
\hline \multicolumn{2}{|l|}{ Variables } & \multicolumn{9}{|c|}{ Substates and probabilities } \\
\hline \multicolumn{2}{|c|}{$\begin{array}{l}\text { Interface management de- } \\
\text { mand }(I M)\end{array}$} & \multicolumn{3}{|l|}{$\left(I M_{1}\right)$} & \multicolumn{3}{|l|}{$\left(I M_{2}\right)$} & \multicolumn{3}{|l|}{$\left(I M_{3}\right)$} \\
\hline \multicolumn{2}{|c|}{$\begin{array}{l}\text { Function allocation between } \\
\text { human-machines and task } \\
\text { design }(F A)\end{array}$} & $\left(F A_{1}\right)$ & $\left(F A_{2}\right)$ & $\left(F A_{3}\right)$ & $\left(F A_{1}\right)$ & $\left(F A_{2}\right)$ & $\left(F A_{3}\right)$ & $\left(F A_{1}\right)$ & $\left(F A_{2}\right)$ & $\left(F A_{3}\right)$ \\
\hline \multirow{3}{*}{$\begin{array}{l}\text { Task inter- } \\
\text { ruption } \\
\text { frequency } \\
(T I)\end{array}$} & $\operatorname{High}\left(T I_{1}\right)$ & 0.95 & 0.74 & 0.58 & 0.47 & 0.28 & 0.17 & 0.30 & 0.14 & 0.10 \\
\hline & $\operatorname{Medium}\left(T I_{2}\right)$ & 0.03 & 0.18 & 0.28 & 0.42 & 0.50 & 0.53 & 0.42 & 0.20 & 0.10 \\
\hline & $\operatorname{Low}\left(T I_{3}\right)$ & 0.02 & 0.08 & 0.14 & 0.11 & 0.22 & 0.30 & 0.28 & 0.66 & 0.80 \\
\hline
\end{tabular}

In order to conduct Bayesian inference, it is necessary to transform fuzzy linguistic probabilities into crisp probabilities. In this paper, fuzzy prior probabilities and fuzzy conditional probabilities are transformed into crisp values by using center of gravity defuzzification technique [17,23].

$$
F_{i}=\frac{\left(u_{i}-l_{i}\right)+\left(m_{i}-l_{i}\right)}{3}+l_{i}
$$

Where $l_{i}, m_{i}$, and $u_{i}$ respectively represent the lower least likely value, the most likely valve, and the upper least likely value. For example, the fuzzy linguistic probability of "severe" for variable "workload transition $(W T)$ " is $(0.06,0.10,0.14)$. It can be transformed into crisp values as follows:

$$
\begin{aligned}
P(W T=\text { severe })= & \frac{(0.14-0.06)+(0.10-0.06)}{3} \\
& +0.06=0.10
\end{aligned}
$$

The transformed values of all the prior probabilities are expressed with bold fonts shown in the Table 1. The conditional probabilities shown in table 2 are all crisp values.

\subsection{Bayesian Inference and Results Analysis}

The inference process is done by calculating the probability distribution of a variable given the observation of other variables, and then updating the probability distributions of unobserved variable. The calculations can be performed manually according to Eq. (1)-(4), or by using software tools like MSBNX [8]. Although Bayesian inference can be used to conduct various types of analysis, this paper mainly discusses predictive inference and diagnostic inference.

\subsubsection{Predictive Inference}

Predictive inference is also called forward inference. It reasons from new information about causes to new beliefs about effects. Supposing that all root nodes obey the initial probability distributions generated by experts, then the probability of each intermediate variable are obtained by using Eq. (2). For example, supposing that "the frequency of task interruption $(T I)$ " is "high $\left(T I_{1}\right)$ ", then the probability of $T I_{1}$ can be computed as follows:

$$
\begin{aligned}
P\left(T I_{1}\right) & =P\left(I M_{1}\right) \times\left[P\left(F A_{1}\right) \times P\left(T I_{1} \mid I M_{1}, F A_{1}\right)\right] \\
& +P\left(I M_{1}\right) \times\left[P\left(F A_{2}\right) \times P\left(T I_{1} \mid I M_{1}, F A_{2}\right)\right] \\
& +P\left(I M_{1}\right) \times\left[P\left(F A_{3}\right) \times P\left(T I_{1} \mid I M_{1}, F A_{3}\right)\right] \\
& +P\left(I M_{2}\right) \times\left[P\left(F A_{1}\right) \times P\left(T I_{1} \mid I M_{2}, F A_{1}\right)\right] \\
& +P\left(I M_{2}\right) \times\left[P\left(F A_{2}\right) \times P\left(T I_{1} \mid I M_{2}, F A_{2}\right)\right] \\
& +P\left(I M_{2}\right) \times\left[P\left(F A_{3}\right) \times P\left(T I_{1} \mid I M_{2}, F A_{3}\right)\right] \\
& +P\left(I M_{3}\right) \times\left[P\left(F A_{1}\right) \times P\left(T I_{1} \mid I M_{3}, F A_{1}\right)\right] \\
& +P\left(I M_{3}\right) \times\left[P\left(F A_{2}\right) \times P\left(T I_{1} \mid I M_{3}, F A_{2}\right)\right] \\
& +P\left(I M_{3}\right) \times\left[P\left(F A_{3}\right) \times P\left(T I_{1} \mid I M_{3}, F A_{3}\right)\right] \\
& =0.12 \times(0.04 \times 0.95+0.54 \times 0.74+0.42 \times 0.58) \\
& +0.87 \times(0.04 \times 0.47+0.54 \times 0.28+0.42 \times 0.17) \\
& +0.01 \times(0.04 \times 0.30+0.54 \times 0.14+0.42 \times 0.10) \\
= & 0.081744+0.210018+0.001296=0.293058
\end{aligned}
$$

Similarly, $P\left(\mathrm{TI}_{2}\right)=0.470758, P\left(\mathrm{TI}_{3}\right)=0.236192$.

Assuming that "workload transition" is "severe", "interface management" is "complicated", "function allocation" is "inappropriate", "automation trust level" is "over-trust", "information overload" is "serious", "keyhole effect" is "severe", "levels of complexity" is "high", and "knowledge degradation" is "severe", then through predictive inference, we can 
Table 3

The change rates between the prior probabilities and the posterior probabilities

\begin{tabular}{|c|c|c|c|c|c|c|c|c|}
\hline Variable states & $W T_{1}$ & $I M_{1}$ & $F A_{1}$ & $A T_{3}$ & $I O_{1}$ & $K E_{1}$ & $L C_{1}$ & $K D_{1}$ \\
\hline Prior probabilities & 0.10 & 0.12 & 0.04 & 0.10 & 0.08 & 0.12 & 0.10 & 0.10 \\
\hline Posterior probabilities & 0.109356 & 0.134335 & 0.040412 & 0.106307 & 0.083891 & 0.128383 & 0.106183 & 0.105683 \\
\hline Change rates $(\%)$ & 9.356 & 11.945 & 1.031 & 6.307 & 4.864 & 6.9858 & 6.183 & 5.683 \\
\hline
\end{tabular}

get the probability of "cognitive reliability $(C R)$ ", $P\left(C R_{1}=" l o w "\right)=0.8227, P\left(C R_{2}=\right.$ "medi um" $)=0.1606$, $P\left(C R_{3}="\right.$ high" $)=0.0167$.

\subsubsection{Diagnostic inference}

Diagnostic inference is also called backward inferences. It reasons from symptoms or effects to its causes. The objective of diagnostic inference is to calculate the occurrence likelihood of some causes given certain consequences. Supposing it is observed that "low level of cognitive reliability $\left(C R_{l}\right)$ " occurs, then according to Eq. (3)-(4), the probabilities of its causes "interface management (IM)" can be calculated as follows:

$$
\begin{aligned}
P(I M= & \left.I M_{1} \mid C R=C R_{1}\right)=\frac{\sum_{U \backslash\left\{M_{1}\right\}} P\left(U, C R_{1}\right)}{P\left(C R_{1}\right)} \\
\sum_{U \backslash\left\{M_{1}\right\}} & P\left(U, C R_{1}\right)=\sum_{U \backslash\left\{M_{1}\right\}} P(T I \mid I M, F A) \times \\
& P(C G \mid I M, T I) \times P(T P \mid I M, T I) \times \\
& P(C G \mid C W, T P, W T) \times P(I A \mid A T, I O, K E) \times \\
& P(M S \mid L C, K D) \times P\left(C R_{1} \mid I A, C G, M S\right) \times \\
& P(I M) \times P(F A) \times P(W T) \times P(A T) \times P(I O) \times \\
& P(K E) \times P(L C) \times P(K D)
\end{aligned}
$$

As every variable has three possible substates, the calculation process is a bit complicated. Here, the MSBNX software is used to implement diagnostic inference. As shown on row 3 and column 3 in Table

3, $P\left(I M=I M_{1} \mid C R=C R_{1}\right)=0.134335$, Similarly, the occurrence probabilities of other causes for $C R_{I}$ can be obtained. The results are specified in the third row of the Table 3.

Comparing the posterior probabilities of root nodes variables with the prior probabilities, the change rates about the occurrence likelihood of each root variable can be seen obviously. As shown in Table 3, the change rates in "interface management" and "workload transition" are more significant than other variables. Once the "cognitive reliability level $(C R)$ " is "low", it is more likely that the "interface management" related errors are the main causes.

\section{Conclusions}

The introduction of computer and digital technologies into NPPs may negatively impact human cognition and behavior. To mitigate the negative consequences imposed by digitalization, there is a need for revealing the nature of these human performance issues. According to the resource-limited and datalimited theory, typical human factors issues which may degrade operators' cognitive performance are evaluated. The results show that the "interface management tasks" exert the greatest impact upon operators' cognitive reliability. The "workload transition" comes next. Other issues such as "keyhole effect", "automation over-trust", and "complexity of technical systems" also have considerable effects on operators.

The investigation and analysis of these human factors issues are helpful to gaining insights for taking probable measures to enhance human reliability. For instance, to minimize the adverse effects of "interface management" and "workload transition", it is crucial to provide special training about multitask management skills and workload regulation strategies. Besides, the MCRs designers should not base only on the capability of technologies, they must pay more attention to the human factors issues, and need to continually modify or improve the system designs through applications of cognitive ergonomics.

\section{Acknowledgements}

The paper is supported in part by National Nature Science Foundation (No. 70873040, 71071051) of China, and Research Projects of Ling Dong Nuclear Power Company Ltd. (No. KR70543 ) of China. Also, 
we are deeply indebted to all the operators who shared their precious insights.

\section{References}

[1] Corragio, L. (1990). Deleterious Effect of Intermittent Interruptions on the Task Performance on Knowledge Workers: a Laboratory Investigation. Unpublished Doctorial Dissertation, University of Arizona.

[2] Dodhia, R. M., \& Dismukes, R. k. (2008). Interruptions create prospective memory tasks. Applied Cognitive Psychology, 22, $1-17$.

[3] Goldberg, R. A., \& Stewart, M. R. (1980). Memory overload or expectancy effect? 'Hysteresis' Revisted. Ergonomics, 23, 1173-1178.

[4] Hockey, G. R. J. (1997). Compensatory control in the regulation of human performance under stress and high workload: A cognitive-energetical framework. Biological Psychology, 45, 73-93.

[5] Jha, P. D, Bisantz, A. M., \& Drury, C. G. (2009). Air traffic controllers' performance in advanced air traffic management systems: Part II Workload and trust. The Journal of Air Traffic Control, 51, 46-52.

[6] Judea, P. (Ed). (2009). Causality: models, reasoning, and inference. Cambridge UK: Cambridge University Press.

[7] Jensen, F. V. (Ed). (1996). An introduction to Bayesian Networks. Berlin: Springer-Verlag.

[8] Kadie, C. M., Hovel, D., \& Horvitz, D. (2001). MSBNX: a component-centric toolkit for modeling and inference with Bayesian networks. Microsoft research technical report MSRTR-2001-67.

[9] Monsell, S. (2003). Task switching. Trends in Cognitive Sciences, 7, 134-140.

[10]Norman, D. A., \& Bobrow, D. G. (1975). On data-limited and resource-limited processes. Cognitive Psychology, 7, 44-64.

[11] O'Hara, J. M., Stubler, W. F., \& Higgins, J. C. (2002). Human Factors Evaluation of Hybrid Human-System Interfaces in Nuclear Power Plants. In Charlton, \& T. G. O'Brien(Eds.), Handbook of Human Factors Testing and Evaluation (pp. 363386). Mahwah: CRC Press.

[12] Orasanu, J. (1997). Stress and naturalistic decision making: Strengthening the weak links. In R. Flin, E. Sales, M. Strub, and L. Martin (Eds.), Decision-making under stress: Emerging themes and applications (pp. 43-66). Brookfield, VT: Ashgate Publishing Company.

[13] O'Hara, J. M, \& Brown, W.S. (2002). The effects of interface management tasks on crew performance and safety in complex, computer-based systems. NUREG/CR-6633. Washington, D.C: U.S. Nuclear Regulatory Commission.

[14] Parasuraman, R., \& Manzey, D. (2010). Complacency and bias in human use of automation: an attentional integration. Human Factors, 52, 381-410.

[15]Reason, J. (1990). Human error. Cambridge: Cambridge University Press.

[16] Rasmussen, J. (1986). Information processing and humanmachine interaction: An approach to cognitive engineering. New York, NY: North-Holland.

[17]Ross, T. J. (Ed). (2004). Fuzzy logic with engineering applications. Chichester: John Wiley \& Sons Ltd.

[18] Sheridan, T. (1980). Computer control and human alienation. Technology Review, 10, 61-73.
[19] Sarter, N., Woods, D. \& Billings, C. (1997). Automation surprises. In G. Salvendy, (Ed.) Handbook of human factors and ergonomics, 2nd ed. (pp.1926-1943). New York:Wiley.

[20] Ungar, N. R., Matthews, G. \& Warm, J. S. (2005). Demand transitions and tracking performance efficiency: Structural and strategic models. Paper presented at the HumanFactors Society $49^{\text {th }}$ Annual Meeting. Santa Monica.

[21] Woods, D., Roth, E, \& Stubler, W. (1990). Navigating through large display networks in dynamic control applications. Paper presented at the Human Factors Society $34^{\text {th }}$ Annual Meeting. Santa Monica.

[22]Wickens, C. D. (2008). Multiple Resources and Mental Workload. Hum Factors, 50, 449-455.

[23]Yang, L. B., \& Gao, Y. Y. (2004). Principle and Application of Fuzzy Math. Guangzhou: South China University of Technology Press (in Chinese). 